\title{
Data Mining in Schizophrenia Research - Preliminary Analysis
}

\author{
Stefan Arnborg ${ }^{1,2}$, Ingrid Agartz ${ }^{3}$, Håkan Hall ${ }^{3}$, Erik Jönsson ${ }^{3}$, \\ Anna Sillén ${ }^{4}$, and Göran Sedvall ${ }^{3}$ \\ 1 Royal Institute of Technology \\ SE-100 44, Stockholm, Sweden \\ stef an@nada.kth.se \\ 2 Swedish Institute of Computer Science \\ 3 Department of Clinical Neuroscience, Section of Psychiatry, Karolinska Institutet \\ SE-171 76 Solna, Sweden \\ 4 Department of Clinical Neuroscience, Karolinska Institutet \\ SE-171 76 Solna, Sweden
}

\begin{abstract}
We describe methods used and some results in a study of schizophrenia in a population of affected and unaffected participants, called patients and controls. The subjects are characterized by diagnosis, genotype, brain anatomy (MRI), laboratory tests on blood samples, and basic demographic data. The long term goal is to identify the causal chains of processes leading to disease. We describe a number of preliminary findings, which confirm earlier results on deviations of brain tissue volumes in schizophrenia patients, and also indicate new effects that are presently under further investigation. More importantly, we discuss a number of issues in selection of methods from the very large set of tools in data mining and statistics.
\end{abstract}

\section{Introduction}

Mental disorders account for a very significant part of total disability in all societies. In particular, every large human population in all parts of the world shows an incidence of schizophrenia between $0.5 \%$ and $1.3 \%$. As for other mental disorders, the cause of the disease is not known, but it has been statistically confirmed that genetic factors and environmental factors before, during and immediately after birth affect its incidence. There is no treatment that cures the disease. Schizophrenia usually leads to life-long disability at great cost for the affected individuals and their families as well as for society.

The HUBIN[13] multi-project is a set of projects aimed at understanding the mechanisms behind mental disorders and in particular schizophrenia. Despite the statement above that the cause of schizophrenia is not known, there are several current and serious hypotheses[3,18]. These center around the development of the neuronal circuitry before birth and during childhood. This development is assumed to be influenced by factors such as genotype, infections, stress and 
social stimulus. The signs of this process can be seen in clinical journals, neuropsychological and psychiatric assessments, and physiological measurements of brain structure and blood contents.

We will describe preliminary findings, and also how the research questions and data available influenced the selection of data analysis methods. These methods are typically adaptations of known tools in statistics and data mining. In section 2 we outline data acquisition, in section 3 the data analysis strategy. Sections 4 and 5 deal with frequentist association assessment and Bayesian multivariate characterization of collected data, respectively. In section 6 we show how the false discovery rate method was used to focus future collection of genetics data, and in section 7 we describe how supervised and unsupervised classification methods are applied to approach our research questions.

\section{Data Acquisition}

The participants included in the study are affected patients with schizophrenia and controls. Each individual has given written consent to participate as regulated by Karolinska Institutet and the 1964 Helsinki Declaration. Exclusion criteria are several conditions that are known to cause unwanted effects on measured variables, among others organic brain disease and brain trauma. Affected individuals were schizophrenia patients recruited from the northern Stockholm region. The control group was recruited from the same region and matched to the affected group with respect to age and gender. All participants underwent interview by an experienced psychiatrist to confirm schizophrenia in the affected group and absence of mental disorders in the control group.

For a set of genes believed to be important for systems disturbed in persons developing schizophrenia, most of the participants were genotyped using single nucleotide polymorphisms (SNP:s). This characterization was obtained using the pyrosequencing method[2].

Participants were investigated in an MR scanner using a standard protocol giving a resolution of $1.5 \mathrm{~mm}$. This protocol admits reliable discrimination of the main brain tissues grey and white matter and cerebro-spinal fluid (CSF), as well as other tissue or fluid like venous blood. Volumes of specific tissues/fluids in regions of interest were obtained by weighted voxel counting. A more detailed description of MR data acquisition can be found in [1].

Blood samples were obtained in which the concentration of certain substances and metabolites are measured with standard laboratory tests. Standard demographic data were obtained, like gender, month of birth, age, and age at first admittance for psychiatric care for the patients.

The choice of variables is determined by current medical hypotheses held by researchers and the possibility of obtaining high quality measurements with reasonable expenditure. Ongoing work aims at collection of detailed psychiatric characterizations, neuropsychological variables and additional genetics information. 


\section{Data Analysis}

The long term goal is to understand the causal chains leading to the disease. It is believed that associations in the data can give important clues. Such clues are then used to determine new data acquisition strategies to confirm preliminary findings and hypotheses.

The most voluminous part of the data set used in this investigation is structural MRI information. The MR scans were converted to $3 \mathrm{D}$ images and processed by the BRAINS software developed at University of Iowa[4,21], to make possible comparisons of corresponding anatomical brain regions in different subjects. This is necessary because of the large individual variations in brain size and shape. The volume of each tissue or fluid type is obtained in a number of regions, like the frontal, temporal, parietal, occipital, subcortical, brainstem and ventricular regions. A number of anatomically distinguishable regions in the vermis of cerebellum (posterior inferior, posterior superior, and anterior vermis) and the cerebellar hemisphere were manually traced (because we have no means to identify them automatically) and measured (total tissue volume only). The reason for including the vermis region is that it is involved in control of eye movements, which are atypical for persons with schizophrenia. The vermis data have been previously analyzed for a limited number of male participants [17]. In the data used here, there are 144 participants, 63 affected and 81 controls, with 30 brain region variables given both in absolute value $(\mathrm{ml})$ and relative to intracranial volume, six summary brain size measures (total volume of discriminated tissue/fluid types), 5 manually measured cerebellar volumes (with absolute and relative values), 58 blood test variables, 20 genetic (SNP) variables (all except 8 were unfortunately uninformative in the sense that almost all participants had the same type), 8 demographic variables, making altogether 144 variables. For some of these there are missing values, which can be regarded as missing completely at random.

The ultimate goal of schizophrenia research is to explain the disease with its large psychiatric and physiological diversity, and to find a good treatment. A more immediate goal is to find tentative answers to the following questions:

- How can causal chains leading to disease be found from our observational data?

- Is it possible to predict the diagnosis and a persons psychiatric conditions from physiological data?

- Do the categorizations used by psychiatrists correspond to recognizable classes for physiological variables?

We have experimented with many available data mining approaches applicable for our type of data, and some useful experiences can be reported. The standard methods described in textbooks are often not immediately applicable, but a method selection and adaptation is called for depending both on the questions to which the answers are sought and on the detailed characteristics of the data. There are many effects of great interest that are very weak or possibly only 
noise in our data, so statistical significance concepts are very important. On the other hand, once an effect has been considered significant and interesting, the best way to communicate the nature of the effect is typically as a graph set graphical models or scatter plots.

There are recently developed methods to find causal links in observational data sets, usually based on the identifiability of arc directions in directed graphical models. Testing our data against a number of different such methods[12], it turned out that in several instances the participants age, month of birth and genotype came out as caused by the phenotype, e.g., the size of a part of the brain would have been a cause of the persons DNA variant. This is highly unlikely to be correct, since the genotype is determined at time of conception, before the development of the individual starts. This finding confirms our belief that the variables presently measured do not include all important information needed to ultimately explain the disease. Our study is thus at this stage oriented somewhat humbly to finding fragments of the processes leading to disease.

\section{Association Tests Based on Randomizations}

The general test method is as follows: We investigate the null hypothesis that the diagnosis was determined at random after the variables were measured. A test statistic was chosen giving a 'difference' between affected and controls, and its value for the data was compared to the cumulative distribution of the test statistics from many random assignments of the diagnosis (in the same proportion as in the original data[10]). The $p$-value obtained is the proportion of more extreme test statistics occurring in the randomized data. As test statistics for single variables we chose the difference between affected and controls in mean and variance. For pairs of variables we normalize the variables and find the angle between the directions, for patients and for controls, of largest variation. The absolute value of the cosine of this angle is used as test statistic.

\section{Multiple Comparison Considerations}

The $p$-values obtained show what the significance would be if the corresponding test was the only one performed, it being customary to declare an effect significant if the $p$-value is below $1 \%$ or $5 \%$ depending on circumstances. However, since many variables and variable pairs were tested, one would expect our tables of significant effects to contain hundreds of spurious entries - even if there were no real effects.

In family-wise error control (FWE[15]), one controls the probability of finding at least one erroneous rejection. A Bonferroni correction divides the desired significance, say $5 \%$, with the number of tests made, and the $p$-values below this value are stated as significant. More sophisticated approaches are possible.

A recent proposal is the control of false discovery rate[6]. Here we are only concerned that the rate (fraction) of false rejections is below a given level. If this rate is set to $5 \%$, it means that of the rejected null hypotheses, on the average no 
more than $5 \%$ are falsely rejected. It was shown that if the tests are independent or positively correlated in a certain sense, one should truncate the rejection list at element $k$ where $k=\max \left\{i: p_{i} \leq q i / m\right\}, m$ is the number of tests and $p_{i}$ is the ordered list of $p$-values. This cut-off rule will be denoted FDRi.

If we do not know how the tests are correlated, it was also shown in [7] that the cut-off value is safe if it is changed from $q i / m$ to $q i /\left(m H_{m}\right)$, where $H_{m}=$ $\sum_{i=1}^{m} 1 / i$. This rule is denoted FDRd. The most obvious correlations induced by the testing in our application satisfy the criterion of positive (monotone) correlation of [7].

Table 1. Significant associations at $5 \%$ with different corrections for multiple testing

\begin{tabular}{lrrrrr} 
& \multicolumn{6}{c}{$m$ Bonf FDRi FDRd no correction } \\
\hline mean & 95 & 28 & 52 & 34 & 56 \\
variance & 95 & 25 & 28 & 26 & 37 \\
angle & 4371 & 53 & 412 & 126 & 723
\end{tabular}

The result of applying various FWE and FDR corrections at $5 \%$ are shown in table 1 . The conclusion is that there is most likely a large number of dependencies among the variables - many more than those found significant above - and the pattern is apparently not explainable by simple models. In application terms, one could say that the disease interacts globally with the development of the brain and permeates into every corner of it. In order to obtain a reasonable amount of clues, we must obviously consider how to find the most important effects. This is a common concern analyzing large and disparate statistical data sets obtainable with modern technology. It has been proposed that maybe Bayes factors are better indicators than $p$-values[16] of effects. The question is not settled, but let us try the Bayesian method and see what we get.

\section{Bayesian Association Determination}

The Bayesian paradigm does not work by rejecting a null hypothesis, but compares two or more specific hypotheses. In our case, hypotheses are compared for each possible association, and the relative support the data give them are summarized as Bayes factors for one against the rest. We have not given detailed prior probabilities to the hypotheses. We can check for multiple testing effects by introducing costs for the two types of error possible. This will have exactly the same effect as a prior probability promoting the null hypothesis. We have penalized for mass testing by giving low prior odds for the dependency hypothesis, so that on the whole our prior information is that on the average only one of the variables should be dependent on the diagnosis. 
The hypotheses in this case are that the same distribution generated the variables for affected and controls, and that two different distributions generated them, respectively. As distribution family we take piece-wise constant functions, which translates to discretization of the variables. The prior distribution over the family is taken to be a Dirichlet distribution. Then the standard association tests of discrete distributions used e. $g$. in graphical model learning[5,14] are applied. An empirical Bayes approach is used, where the granularity is chosen to give a sufficient number of points in each discretization level.

\section{Bayesian Association Models}

For a chosen discretization, a variable will be described as an occurrence vector $\left(n_{i}\right)_{i=1}^{d}$, where $d$ is the number of levels and $n_{i}$ is the number of values falling in bin $i$. Let $\bar{x}=\left(x_{i}\right)_{i=1}^{d}$ be the probability vector, $x_{i}$ being the probability of a value falling in bin $i$. A Bayesian association test for two variables is a comparison of two hypotheses, one $H_{d}$ in which the variables are jointly generated and one $H_{i}$ in which they are independently generated.

Table 2. Bayesian association (log Bayes factor), variable to diagnosis. Strongly associated variables are brain regions, but also serum triglycerides

\begin{tabular}{ll} 
Variable & $\log (B F)$ \\
\hline rel post sup vermis & 8.08 \\
abs post sup vermis & 7.77 \\
rel temporal CSF & 6.37 \\
abs total vermis & 5.68 \\
rel total vermis & 5.18 \\
abs temporal CSF & 4.29 \\
ratio CSF/grey & 4.25 \\
rel brainstem CSF & 3.41 \\
rel total CSF & 3.27 \\
abs brainstem CSF & 3.08 \\
abs total CSF & 3.06 \\
\hline
\end{tabular}

\begin{tabular}{ll} 
Variable & $\log (B F)$ \\
\hline serum triglycerides & 2.91 \\
rel post inf vermis & 2.78 \\
abs post inf vermis & 2.71 \\
abs ventricular white & 2.55 \\
rel total CSF & 2.35 \\
rel ventricular white & 2.34 \\
abs anterior vermis & 2.32 \\
rel ventricular CSF & 2.24 \\
abs subcortical white & 2.23 \\
abs ventricular CSF & 2.1 \\
rel anterior vermis & 1.89 \\
\hline
\end{tabular}

Table 2 gives the $\log$ Bayes factors, $\log \left(p\left(\bar{n} \mid H_{d}\right) / p\left(\bar{n} \mid H_{i}\right)\right)$, of $H_{d}$ against $H_{i}$ for variables discretized into 5 levels. Assuming the previously mentioned prior, entries with $\log$ Bayes factor above 2 would be deemed significant.

For the co-variation investigation we chose to compare the eight undirected graphical models on triples of variables, one of which is the diagnosis. If the graph described by the complete graph, a triangle, on the three variables has high posterior probability, then this means that the variation of the data cannot be described as resulting from influence of the diagnosis on one of the two variables or as independent influence on both - the association between the variables is different for affected and controls. In figure 1, the left graph represents 

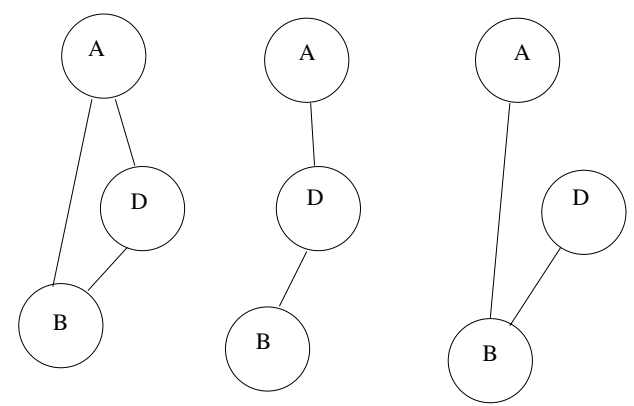

Fig. 1. Graphical models detecting co-variation

the type of co-variation we are looking for. The next graph explains data as the diagnosis $D$ affecting variables $A$ and $B$ separately, whereas the rightmost graph describes a situation where the association between $A$ and $B$ is similar for affected and controls. This method can be generalized to higher order interactions, but we need substantially more data before this becomes meaningful. In both frequentist and Bayesian pairwise variable association studies, the posterior superior vermis was highly linked via the diagnosis to several other variables. Particularly interesting is the age variable, which is known to be independent (figure 2(b)). For patients the posterior superior vermis is smaller and not dependent on age, whereas for controls it decreases with age. The hypothesis that the change in vermis size develops before outbreak is natural and made even more likely by not being visibly dependent of medication and length of the disease period.

Bayesian association estimates are the basis for graphical models giving an overview of co-variation of variable sets. Based on the strength of pairwise variable associations, decomposable graphical models were obtained from the data matrix. For the matrix containing demographic, physiology and automatically measured white, gray and CSF volumes, and genotype, the central part of the
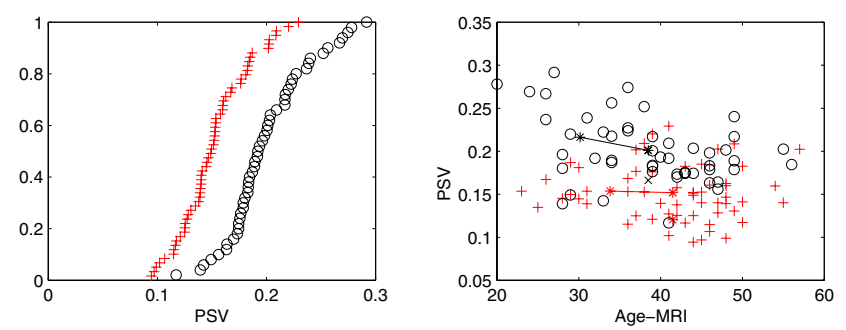

Fig. 2. (a) Empirical cumulative distributions for posterior superior vermis, + : affected, o : controls. (b) Scatter plot of association in angle of principal directions of variation, $\log p \approx-3$ 
model was found to be as shown in figure 3(a). In words, the diagnosis is most distinctly, in statistical terms, associated with the CSF volumes in the brainstem and temporal regions.

Even more closely associated are the vermis regions that were measured manually (volumes only). When the vermis variables are also included, the model looks like figure 3(b). The position of the temporal CSF variable in the diagram suggests that a brain region affected similarly to the vermis can be located in the temporal boxes.
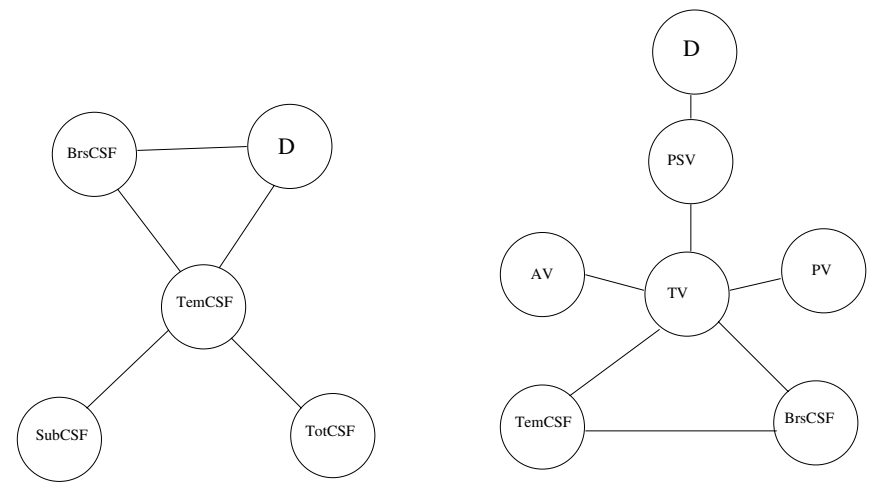

Fig. 3. Graphical models, neighborhoods of Diagnosis (D) - (a)grey/white/CSF volumes; (b) Vermis volumes added

\section{Genetics Data}

The genetic variables were not strongly associated to phenotype variables measured. This does not mean that they are uninteresting. The basic machinery of biological systems is run by proteins, and the genes are blueprints of these proteins. The different variations, alleles, of a gene result in small variations in the proteins they produce. For single gene diseases there is often a single variation of the protein that immediately leads to the disease, but for multiple gene diseases, to which schizophrenia apparently belongs, there is a whole family of genes with variants that have small effects on the disposition for disease, in other words they push the whole organisms development slightly in the direction where the etiology of disease can begin to unfold. The complexities of these processes are overwhelming, and although a large amount of knowledge has been accumulated over a few decades, it is fair to say that even more is presently unknown.

The SNP genotyping separates the alleles of a gene into two classes, and these two classes can be slightly different in function of the protein and its effect on the development of an individual. By finding associations between genotype 
and other variables, information about the role of the corresponding protein and its metabolites in development can be extracted. Because of the weakness of the statistical signals, genetics data must be examined with the most powerful - but sound - statistical methods available.

The informative genes and their polymorphisms measured for the population are shown in table 3.

Table 3. Genes with informative SNP:s

\begin{tabular}{lllll} 
Gene & SNP type & polym & informative & function \\
\hline DBH & Ala55Ser & G/T & 98240 & dopamine beta-hydroxylase \\
DRD2 & Ser311Cys G/C & 11840 & dopamine receptor D2 \\
DRD3 & Ser9Gly & A/G & 495914 & dopamine receptor D3 \\
HTR5A & Pro15Ser & C/T & 109112 & serotonin receptor 5A \\
NPY & Leu7Pro & T/C & 17114 & neuropeptide Y \\
SLC6A4 ins/del & S/L & 206042 & serotonin transporter \\
BDNF & Val66Met & A/G & 53780 & brain derived neurotrophic factor
\end{tabular}

A Bayesian comparison with the diagnosis variable speaks weakly in favor of independence between genotype and diagnosis for all polymorphisms available. The same is true when using Fishers exact test. But we have also many other variables related to brain development. Testing the genes against all variables, $63 p$-values below $5 \%$ were found. However, applying the FDRd or FDRi correction on $5 \%$ false rejection rate, none of these survive. It is somewhat remarkable, however, that 30 small $p$-values are related to the polymorphism in the BDNF gene. There is thus ground for the suspicion that this polymorphism has a an influence on brain development that will probably identifiable in MR images with slightly more cases. It is possible to state this influence in statistical terms: in the FDR sense, on the average $80 \%$ of 30 variables are affected by this polymorphism. Among these variables are the manually traced posterior inferior vermis, gray matter in the frontal, parietal, subcortical, temporal and ventricular regions. Interestingly, a Bayesian modeling with a linear model and using SSVS variable selection of [11] does not show this effect unless the noise level is forced down unreasonably. This can be explained by the imperfect fit to the linear model assumptions. Thus, in this case the randomization and FDR methods are instrumental because they give easily a justifiable inference that points to a subset of the included genes as promising, and allows further data collection to concentrate on these.

Some of the strongest associations found are shown in figure 4. In summary, the current genetics data show that there are likely genetic dependencies of variables, but the statistical power is not yet adequate to identify specific associations with high significance. 

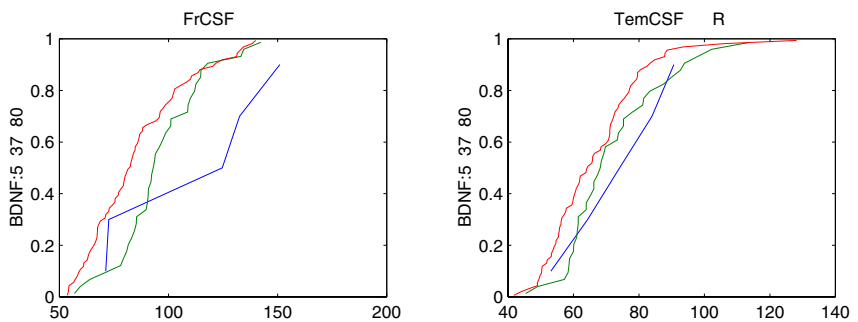

Fig. 4. Possible genetic effects picked out by $p$-values and Bayesian model comparisons. Empirical cumulative distributions of the variable (frontal CSF and temporal CSF) for the three types (A/A, A/G and $G / G)$ of SNP in gene BDNF.

\section{Prediction and Classification Study}

It is a known difficult problem to determine the diagnosis of schizophrenia from physiological variables. The problem is important for the potential of early risk detection in research and for treatment. We checked that this is also the case for our data sets. Supervised classifiers were built using both support vector[9] and decision tree[5] techniques. When trained on random samples of $75 \%$ of the participants and tested on the remaining individuals, a classification accuracy of approximately $78 \%$ was obtained. If only the optimal discriminating single variable, post sup vermis, is used with the same training process, $71 \%$ accuracy is obtained, and it thus has a dominating explanatory power (see figure 2(a)). Another technique that has been useful is unsupervised classification. The interest in this problem is motivated by the possibility of there being several different processes leading to disease with different physiological traces, and for checking against the wide span of symptom sets that develop in different patients. Classifications of schizophrenia patients have usually been performed with cluster analysis paradigms[19]. The AUTOCLASS paradigm tries to find classifications with high probability, under assumptions of particular distributions for the variables within each class. We ran the AUTOCLASS software[8] on a subset of participants and variables without missing values. The variables where assumed to be independent, categorical or normally distributed within each class. AUTOCLASS searches for the classification in the form of a probability mixture with maximal probability of generating the data. In our case, a four class mixture was identified for the population consisting of $42 \%$ affected. The most important variable for the classification was total (absolute) volume of gray matter. One of the classes has mainly $(75 \%)$ controls and a high value for gray matter. The next class has a high proportion of patients $(83 \%)$, somewhat less but dispersed (high variance) gray matter. A third class has the same amount of gray matter, but with low variance and not very discriminated wrt diagnosis $(33 \%$ affected). The final class has low volume of gray matter and $34 \%$ affected. Other classification approaches are presently under investigation. 


\section{Summary and Conclusions}

The application findings reported above are interesting although the ultimate explanation of schizophrenia seems still far away.

On the methodological side, it is clear that the data mining philosophy implies making a large number of tests and similar investigations involving both $p$ values, Bayesian model comparisons and visual inspections. We found Bayesian analysis of graphical model sets useful for characterization of multivariate data with obvious interdependencies, whereas randomization and the FDR method was more sensitive for detecting associations between genotype and phenotype. It is also obvious that the methods complement each other - testing is appropriate when the null hypothesis can be precisely formulated and then for any test statistic randomization tests can give a $p$-value, the classical risk that an erroneous conclusion is drawn. But Bayesian model comparison is a very natural and easily implemented method that gives answers also when there is no obvious null hypothesis. Lastly, graphical visualizations are necessary as confirmations of statistical effects.

The strict control of multiple comparison effects can not easily be formalized in typical data mining since many investigations are summarily discarded for lack of obvious effects or lack of interest from application experts. Our method must make an intricate balance between creation of significance and creation of sense - without inventing an implausible story. This is a problem that seems not yet fully addressed, neither in the statistics nor in the data mining communities. Some further development of the $q$-values proposed by Storey[20] might be useful for this purpose

\section{Acknowledgments}

The cerebellum variables included in this study were obtained by Gaku Okugawa. The AUTOCLASS run results were obtained by Can Mert. We acknowledge valuable discussions with Stig Larsson, Tom McNeil, Lars Terenius and Manuela Zamfir. The HUBIN project is funded by the Wallenberg foundation.

\section{References}

1. I. Agartz, V. Magnotta, M. Nordström, G. Okugawa, and G. Sedvall. Reliability and reproducibility of brain tissue volumetry from segmented MR scans. European Archives of Psychiatry and Clinical Neuroscienc, pages 255-261, 2001. 28

2. A. Ahmadian, B. Gharizadeh, A. C. Gustafsson, F. Sterky, P. Nyren, M. Uhlen, and J. Lundeberg. Single-nucleotide polymorphism analysis by pyrosequencing. Analytical Biochemistry, 2000. 28

3. N. C. Andreasen. Linking mind and brain in the study of mental illnesses: a project for a scientific psychopatology. Science, 275:1586-1593, 1997. 27

4. N. C. Andreasen, R. Rajarethinam, T. Cizaldo, S. Arndt, V. W. II Swayze, L. A. Flashman, D. S. O'Leary, J. C. Ehrherdt, and W. T. C. Yuh. Automatic atlas-based volume estimation of human brain regions from MR images. J. Comput. Assist. Tomogr., 20:98-106, 1996. 29 
5. S. Arnborg. A survey of Bayesian data mining. Technical report, 1999. SICS TR T99:08. 32, 36

6. Y. Benjamini and Y. Hochberg. Controlling the false discovery rate: A practical and powerful approach to multiple testing. J. of the Royal statistical Society B, 57:289-300, 1995. 30

7. Y. Benjamini and D. Yekutieli. The control of the false discovery rate in multiple testing under dependency. Technical report, Stanford University, Dept of Statistics, 2001. 31

8. P. Cheeseman and J. Stutz. Bayesian classification (AUTOCLASS): Theory and results. In U. M. Fayyad, G. Piatetsky-Shapiro, P Smyth, and R. Uthurusamy, editors, Advances in Knowledge Discovery and Data Mining. 1995. 36

9. N. Cristianini and J. Shawe-Taylor, editors. Support Vector Machines and other kernel based methods. Cambridge University Press, 2000. 36

10. E. Edgington. Randomization Tests. New York, M. Dekker, 1987. 30

11. E. I. George and R. E. McCulloch. Approaches for bayesian variable selection. Technical report, The Univeristy of Texas, Austin, 1996. 35

12. C. Glymour and G. Cooper, editors. Computation, Causation and Discovery. MIT Press, 1999. 30

13. Håkan Hall, Stig Larsson, and Göran Sedvall. Human brain informatics - HUBIN web site. 1999. http://hubin.org. 27

14. David Heckerman. Bayesian networks for data mining. Data Mining and Knowledge Discovery, 1:79-119, 1997. 32

15. Y. Hochberg. A sharper Bonferroni procedure for multiple tests of significance. Biometrika, 75:800-803, 1988. 30

16. J. I. Marden. Hypothesis testing: From $p$-values to Bayes factors. J. American Statistical Ass., 95:1316-1320, 2000. 31

17. G. Okugawa, G. Sedvall, M. Nordström, N. C. Andreasen, R. Pierson, V. Magnotta, and I. Agartz. Selective reduction of the posterior superior vermis in men with chronic schizophrenia. Schizophrenia Research, (April), 2001. In press. 29

18. G. Sedvall and L. Terenius. In Schizophrenia: Pathophysiological mechanisms. Proceedings of the Nobel Symposium 111(1998) on Schizophrenia. Elsevier, 2000. 27

19. S. R. Sponheim, W. G. Iacono, P. D. Thuras, and M. Beiser. Using biological indices to classify schizophrenia and other psychotic patients. Schizophrenia Research, pages $139-150,2001.36$

20. J. Storey. The false discovery rate: A Bayesian interpretation and the $q$-value. Technical report, Stanford University, Dept of Statistics, 2001. 37

21. R. P. Woods, S. T. Grafton, J. D. Watson, N. L. Sicotte, and J. C. Maziotta. Automated image registration: II. intersubject validation of linear and non-linear models. J. Comput. Assist. Tomogr., 22:155-165, 1998. 29 